\title{
Smoothness Equivalence Properties of Interpolatory Lie Group Subdivision Schemes
}

\author{
Gang XIE * Thomas P.-Y. Yu ${ }^{\dagger}$ \\ March 15, 2007 \\ revised September 15, 2008
}

\begin{abstract}
:
We prove that any interpolatory Lie group subdivision scheme based on combining a linear interpolatory subdivision scheme $\bar{S}$ with the log-exp adaption to Lie group valued data in [8] produces parametrized curves on the Lie group which are as smooth as the smoothness of $\bar{S}-$ no matter how smooth $\bar{S}$ is. We present both an extrinsic proof and an intrinsic proof.

We discuss two variations of our main result. (i) We illustrate how smoothness equivalence can break down in a variant of the original log-exp scheme. (ii) We show that the main result of this paper can be easily extended to a multivariate setting.

Acknowledgments. The work of this research was partially supported by the National Science Foundation grant DMS 0512673. The authors thank Tom Duchamp, William Goh, Greg Naber, Johannes Wallner, Andy Hicks and Arieh Iserles for helpful discussions. The work of this research was partially supported by the National Science Foundation grant DMS 0512673. Part of the results in this paper was reported in the Twelfth International Conference in Approximation Theory held in San Antonio, Texas, USA during March 4-8, 2007.
\end{abstract}

Keywords. Subdivision schemes, Lie groups, Matrix Lie groups, Interpolation, Smoothness equivalence, Breakdown of smoothness equivalence

\footnotetext{
${ }^{*}$ Department of Mathematics, East China University of Science and Technology, Shanghai, China, 200237. Email: gangxie2006@gmail.com.

${ }^{\dagger}$ Department of Mathematics, Drexel University, 3141 Chestnut Street, 206 Korman Center, Philadelphia, PA 19104, U.S.A.. Email: yut@drexel.edu. URL: http://www.math.drexel.edu/ tyu.
} 


\section{Introduction}

Given a set of control points $y_{i}$ on a Lie group $(G, \cdot)$, how would one find a smooth parametrized curve $c(t)$ such that $c(i)=y_{i}$ ? We would like the interpolation method to respect the symmetry of $G$ : if the points $y_{i}$ are shifted to $z_{i}=g \cdot y_{i}$ by an arbitrary $g \in G$, then the interpolating curve of $z_{i}$ given by the same method should be $g \cdot c(t)$.

A natural way to approach this problem is to map the data $\left(y_{i}\right)_{i}$ from the Lie group $G$ to its Lie algebra $\mathfrak{g}$ using the inverse of the exponential map, then apply any standard linear method of interpolation in $\mathfrak{g}$, and then pullback the interpolant to $G$ by the exponential map. See, for example, [7]. This approach works when all the data points $\left(y_{i}\right)_{i}$ are close enough, but fails in a fundamental way when this assumption is violated. This is by and large due to the typical nontrivial topology of $G$, see [9] for a detailed study of the case $G=S O(3)$.

The local and multiscale nature of subdivision methods somehow alleviates the topological obstacle substantially. We shall prove that the subdivision methods studied in this paper provide provably smooth interpolants as long as the data points $\left(y_{i}\right)_{i}$ are locally close. Subdivision techniques are also ideally suited when different level-of-details are desired; and can be deployed to construct nonlinear wavelet-type transforms of manifold valued data, see [8]. For more discussions on these methods, see our earlier paper [14].

An interpolatory subdivision scheme $S$ for Lie group valued data works as follows. Given initial data defined on a uniform grid $y_{0}: \mathbb{Z} \rightarrow G, S$ creates data $y_{j}$ on the dyadic grids $2^{-j} \mathbb{Z}$ recursively by

$$
y_{j+1,2 i}=y_{j, i}, \quad y_{j+1,2 i+1}=R\left(y_{j, i-L}, \cdots, y_{j, i-L+p}\right),
$$

where, in above, $L$ and $p$ are fixed integers (the 'locality factors' of the scheme), and $R$ is a fixed mapping they do not change with either the scale $j$ or location $i$; such a scheme is termed stationary. For simplicity of analysis, we assume that the scheme takes the form

$$
y_{j+1,2 i}=y_{j, i}, \quad y_{j+1,2 i+1}=y_{j, i} \cdot R\left(y_{j, i}^{-1} \cdot y_{j, i-L}, \cdots, y_{j, i}^{-1} \cdot y_{j, i-L+p}\right) .
$$

As such, we can assume that $R\left(y_{0}, \ldots, y_{p}\right)$ is only defined for inputs $y_{k}, 0 \leqslant k \leqslant p$, in a neighborhood of $e$ of the Lie group; and the overall subdivision scheme is meant to be applied to dense enough initial data. Note that the form (1.2) guarantees that the resulted interpolation algorithm is invariant under an arbitrary shift of initial control data.

Many variations can be derived based on (1.1), to 'life on an interval', to non-uniform grids, to Hermite data, to non-stationary schemes, to multivariate settings, to manifolds other than Lie groups [15] etc.. Of course a general subdivision scheme needs not be interpolatory. We focus on (1.1) in this paper, but will show at the end of the paper that there is an easy generalization of our main result to a multivariate setting.

Recall that every Lie group $G$ has a Lie algebra $\mathfrak{g}$ which can be identified with the tangent plane of $G$ at the identity element $e$, so we write $\mathfrak{g}=T_{e} G$. Recall also the exponential map

$$
\exp : \mathfrak{g} \rightarrow G, \quad v \mapsto \phi_{v}(1),
$$

where $\phi_{v}: \mathbb{R} \rightarrow G$ is the unique 1-parameter subgroup of $G$ with $\phi_{v}^{\prime}(0)=v$. While the exp map is defined on the whole of $\mathfrak{g}$, it is typically not injective, and may or may not be surjective. Nevertheless, there is always a neighborhood $U$ of 0 in $\mathfrak{g}$ and a neighborhood $\widetilde{U}$ of $e$ in $G$ such that

$$
\left.\exp \right|_{U}: U \rightarrow \widetilde{U}
$$

is a diffeomorphism. The inverse of this diffeomorphism is called the log map of the Lie group, and we refer to the $\widetilde{U}$ above as $\operatorname{dom}(\log )$ when the context requires. Sometimes we write $\exp _{G}$ and $\log _{G}$ to signify the dependence on $G$.

A specific scheme proposed in [8] is, in our notation here, given by $R=R_{D}$ where

$$
R_{D}\left(x_{0}, \ldots, x_{p}\right)=\exp \left(\sum_{\ell=0}^{p} a_{\ell} \log \left(x_{\ell}\right)\right),
$$


where the $a_{\ell}$ 's are specific weights chosen according to the mask of a Deslauriers-Dubuc interpolatory subdivision scheme $[2,1]$. A different scheme is proposed in [13], and the associated $R=R_{W}$ is given by:

$$
R_{W}\left(x_{0}, \ldots, x_{p}\right)=\exp \left(\sum_{\ell=0}^{p-1} b_{\ell} \log \left(x_{\ell}^{-1} x_{\ell+1}\right)\right)
$$

where $\left(b_{\ell}\right)_{\ell=0}^{p-1}$ is derived from $\left(w_{\ell}\right)_{\ell=0}^{p}$ via (3.2); see Section 3.1.

The goal of this paper is two-folded. (I) We prove that the nonlinear scheme based on (1.3) is as smooth as the underlying linear interpolatory subdivision scheme. (II) We explore to what extent smoothness equivalence holds for the nonlinear scheme based on (1.4); we illustrate a breakdown phenomenon of smoothness equivalence for this scheme.

\section{$2 \quad$ Main Result}

We say that a subdivision scheme $S$ is $C^{\sigma}$ smooth if for any dense enough initial data, the limit function $f: \mathbb{R} \rightarrow G$ has a Hölder regularity with exponent $\sigma$. Here, Hölder regularity is defined based on the differentiable structure of $G$. Following [14], we also denote by $s_{\infty}(S)$ the critical Hölder regularity of the scheme $S$. We say that a subdivision scheme $S$ is convergent if $s_{\infty}(S)>0$.

The main result of this paper is similar to those in our earlier paper [14].

Theorem 2.1. Let $G$ be any finitely dimensional Lie group. If $S$ is the subdivision scheme in $G$ defined by (1.1) with $R=R_{D}$, and $\bar{S}$ is the underlying linear subdivision scheme. Then $s_{\infty}(S) \geqslant s_{\infty}(\bar{S})$.

We shall present two different proofs of this result. The first proof is extrinsic in nature, whereas the second is intrinsic in nature. The extrinsic proof is perhaps more concrete to some readers, at least the part which deals with the case of $G$ being a closed matrix group. Ultimately, the intrinsic proof is much more natural. On the other hand, we found it interesting that essentially the same proximity bounds can be derived in both the extrinsic and the intrinsic setups. Yet another extrinsic proof can be found in the recent manuscript [4].

Section 2.2 and Section 2.3 are independent of each other. Modulo the notation $\mathcal{N}(j, i)$ defined in the paragraph around (2.14) in Section 2.2.1, Section 2.3 is self-contained.

\subsection{Proximity implies smoothness}

For both the extrinsic and intrinsic proofs, we need the following theorem.

For $j \in \mathbb{N}$, let

$$
\Gamma_{j}:=\left\{\gamma=\left(\gamma_{1}, \ldots, \gamma_{j}\right) \mid \gamma_{i} \in \mathbb{N} \cup\{0\}, \sum_{i=1}^{j} \gamma_{i} \geqslant 2, \quad \sum_{i=1}^{j} i \gamma_{i} \geqslant j+1\right\} .
$$

For any sequence $x: \mathbb{Z} \rightarrow \mathbb{R}^{n}$, let

$$
\Omega_{j}(x):=\sum_{\gamma \in \Gamma_{j}}|\Delta x|_{\infty}^{\gamma_{1}} \cdots\left|\Delta^{j} x\right|_{\infty}^{\gamma_{j}}
$$

Theorem 2.2. Let $S$ be a subdivision operator and $\bar{S}$ be a linear subdivision operator. Assume that both $S$ and $\bar{S}$ are interpolatory and have the same dilation factor. Let $k$ be an integer greater than $s_{\infty}(\bar{S})$. Assume that, for any $j=1, \ldots, k$,

$$
|S x-\bar{S} x|_{\infty}=O\left(\Omega_{j}(x)\right)
$$

holds true for any dense enough sequence $x: \mathbb{Z} \rightarrow \mathbb{R}^{N}$, then $s_{\infty}(S) \geqslant s_{\infty}(\bar{S})$.

The proof is just a trivial modification of that of [14, Theorem 3.8]; the argument there has a 'bootstrapping' flavor which relies quite heavily on the interpolatory assumption. There is an extension of this theorem in [15] which handles subdivision schemes which are just $L^{\infty}$-stable but not necessarily interpolatory. 


\subsection{An Extrinsic Proof}

In this section, let $G$ be a matrix (Lie) group. A matrix (Lie) group is defined, e.g. as in [10] and [11], to be a subgroup $G$ of $G L(n)$, and also a Lie group by itself for some $C^{\infty}$ structure which makes the inclusion map $\mathfrak{i}: G \rightarrow \mathbb{R}^{n \times n}$ an immersion. Under this definition, $G$ needs not be closed, and, hence, also needs not be a regularly imbedded submanifold of $\mathbb{R}^{n \times n}$. For a matrix group, the exp is given by the series $\exp (X)=\sum_{k=0}^{\infty} \frac{X^{k}}{k !}$. When $X$ is close enough to $\mathbb{I}$ (precisely, when the spectral radius of $X-\mathbb{I}<1$ ), then

$$
\log (X)=\sum_{k=1}^{\infty} \frac{(-1)^{k-1}(X-\mathbb{I})^{k}}{k} .
$$

(It is not true in general that every $X \in \operatorname{dom}\left(\log _{G}\right)$ is close enough to $\mathbb{I}$ for the above log series to converge. As a simple example, if $G=G L(1)=\mathbb{R} \backslash\{0\}, \mathfrak{g}=g l(1)=\mathbb{R}$, then $\operatorname{dom}\left(\log _{G}\right)=\mathbb{R}^{+}$, but (2.1) only holds for $0<X<2$.)

Let $y: \mathbb{Z} \rightarrow G$ be a sequence such that $y_{i}^{-1} y_{i+\ell} \in \operatorname{dom}\left(\log _{G}\right)$ and also $y_{i}^{-1} y_{i+\ell}$ stays within the radius of convergence of the $\log$ series for all $i \in \mathbb{Z}$ and all $\ell$ in the support of the mask $\left(w_{\ell}\right)$. Assume that this mask is supported at $[-L,-L+p]$. Then

$$
S y_{2 i+1}:=y_{i} \exp \left(\sum_{\ell=-L}^{-L+p} w_{\ell} \log \left(y_{i}^{-1} y_{i+\ell}\right)\right)
$$

is well-defined. In fact it can be shown that if $s_{\infty}(\bar{S})>0$, and $y$ is dense enough, then $S^{j} y$ is well-defined for all $j>0$. We shall prove this claim, along with a quantification of 'dense enough', in Section 2.3.

Next, we also have the linear interpolatory scheme

$$
\bar{S} y_{2 i+1}=\sum_{\ell=-L}^{-L+p} w_{\ell} y_{i+\ell}=y_{i} \sum_{\ell=-L}^{-L+p} w_{\ell} y_{i}^{-1} y_{i+\ell} .
$$

If we write $d_{\ell}=y_{i}^{-1} y_{i+\ell-L}-\mathbb{I}, a_{\ell}=w_{\ell-L}$ and

$$
s_{k}=\sum_{\ell=0}^{p} a_{\ell} d_{\ell}^{k},
$$

then

$$
\begin{aligned}
y_{i}^{-1}\left(S y_{2 i+1}-\bar{S} y_{2 i+1}\right)= & \exp \left(\sum_{\ell=0}^{p} a_{\ell}\left(d_{\ell}-d_{\ell}^{2} / 2+d_{\ell}^{3} / 3 \mp \cdots\right)\right)-\sum_{\ell=0}^{p} a_{\ell}\left(d_{\ell}+\mathbb{I}\right) \\
= & \exp \left(s_{1}-s_{2} / 2+s_{3} / 3 \mp \cdots\right)-\mathbb{I}-s_{1} \\
= & \left(-\frac{1}{2} s_{2}+\frac{1}{3} s_{3} \mp \cdots\right)+\frac{1}{2 !}\left(s_{1}-\frac{1}{2} s_{2}+\frac{1}{3} s_{3} \mp \cdots\right)^{2}+ \\
& \frac{1}{3 !}\left(s_{1}-\frac{1}{2} s_{2}+\frac{1}{3} s_{3} \mp \cdots\right)^{3}+\frac{1}{4 !}\left(s_{1}-\frac{1}{2} s_{2}+\frac{1}{3} s_{3} \mp \cdots\right)^{4}+\cdots .
\end{aligned}
$$

Note that the cancelation of the first-order terms relies on the first sum rule $\sum a_{\ell}=1$.

The right-hand side of (2.5) consists of terms of the form $s_{k_{1}} \cdots s_{k_{m}}$ when we expand the powers. We define the degree of such a term to be $\sum_{i=1}^{m} k_{i}$; and we shall expand out all the powers in $(2.5)$ and then order the terms using their degrees. Collecting the terms with degree 2, we get

$$
T_{2}:=-s_{2} / 2+s_{1}^{2} / 2
$$

for degree 3 , we get $T_{3}:=s_{3} / 3-s_{1} s_{2} / 4-s_{2} s_{1} / 4+s_{1}^{3} / 6$. In general, if we collect the terms with a degree $k \geqslant 2$, we get

$$
\begin{aligned}
T_{k} & :=\sum_{1 \leqslant m \leqslant k} \sum_{i_{1}+\cdots+i_{m}=k} \frac{(-1)^{k-m}}{m ! i_{1} \cdots i_{m}} s_{i_{1}} \cdots s_{i_{m}} \\
& =\sum_{\substack{1 \leqslant m \leqslant k \\
1 \\
i_{1}+\cdots+i_{m}=k}} \frac{(-1)^{k-m}}{m ! i_{1} \cdots i_{m}}\left(\sum_{\ell} a_{\ell} d_{\ell}^{i_{1}}\right) \cdots\left(\sum_{\ell} a_{\ell} d_{\ell}^{i_{m}}\right) .
\end{aligned}
$$


To conclude, we have

$$
y_{i}^{-1}\left(S y_{2 i+1}-\bar{S} y_{2 i+1}\right)=\sum_{k=2}^{\infty} T_{k} .
$$

The convergence is absolute with respect to any matrix norm.

Let $D_{\ell}$ be the $\ell$-th order difference of $d_{0}, \ldots, d_{\ell}$, i.e.

$$
D_{\ell}:=\sum_{j=0}^{\ell}(-1)^{\ell-j}\left(\begin{array}{l}
\ell \\
j
\end{array}\right) d_{j}
$$

We can recover $d_{0}, \ldots, d_{p}$ from $D_{0}, \ldots, D_{p}$ by

$$
d_{\ell}=\sum_{j=0}^{\ell}\left(\begin{array}{l}
\ell \\
j
\end{array}\right) D_{j}
$$

Substituting (2.9) into (2.6) and expanding out everything, we can rewrite (2.6) as a linear combination of terms of the form $D_{j_{1}} \cdots D_{j_{k}}$ where $j_{1}, \ldots, j_{k}$ can be any integers between 0 and $p$, i.e.

$$
\begin{aligned}
T_{k} & =\sum_{\substack{1 \leqslant m \leqslant k \\
i_{1}+\cdots+i_{m}=k}} \frac{(-1)^{k-m}}{m ! i_{1} \cdots i_{m}}\left(\sum_{\ell} a_{\ell}\left[\sum_{j=0}^{\ell}\left(\begin{array}{l}
\ell \\
j
\end{array}\right) D_{j}\right]^{i_{1}}\right) \cdots\left(\sum_{\ell} a_{\ell}\left[\sum_{j=0}^{\ell}\left(\begin{array}{l}
\ell \\
j
\end{array}\right) D_{j}\right]^{i_{m}}\right) \\
& =\sum_{0 \leqslant j_{1}, \ldots, j_{k} \leqslant p} c_{j_{1} \cdots j_{k}} D_{j_{1}} \cdots D_{j_{k}} .
\end{aligned}
$$

Note that

and

$$
D_{j_{1}} \cdots D_{j_{k}}=\left(D_{j_{1}} \cdots D_{j_{i_{1}}}\right)\left(D_{j_{i_{1}+1}} \cdots D_{j_{i_{1}+i_{2}}}\right) \cdots\left(D_{j_{i_{1}+\cdots+i_{m-1}+1}} \cdots D_{j_{k}}\right)
$$

$$
c_{j_{1} \cdots j_{k}}=\sum_{\substack{1 \leqslant m \leqslant k \\
i_{1}+\cdots+i_{m}=k}} \frac{(-1)^{k-m}}{m ! i_{1} \cdots i_{m}}\left[\sum_{\ell=0}^{p} a_{\ell}\left(\begin{array}{c}
\ell \\
j_{1}
\end{array}\right) \cdots\left(\begin{array}{c}
\ell \\
j_{i_{1}}
\end{array}\right)\right] \cdots\left[\sum_{\ell=0}^{p} a_{\ell}\left(\begin{array}{c}
\ell \\
j_{i_{1}+\cdots+i_{m-1}+1}
\end{array}\right) \cdots\left(\begin{array}{c}
\ell \\
j_{k}
\end{array}\right)\right] .
$$

This coefficient may look horrendous, but it has the following nice property.

Proposition 2.3. Let $k \geqslant 2,0 \leqslant j_{1}, \ldots, j_{k} \leqslant p$. If the mask $\left(a_{\ell}\right)_{\ell=0}^{p}$ reproduces $\Pi_{\bar{p}}$, then

$$
j_{1}+\cdots+j_{k} \leqslant \bar{p} \Longrightarrow c_{j_{1} \cdots j_{k}}=0 .
$$

This proposition can be easily proved once we establish the following identity:

Lemma 2.4. For any $B \in \mathbb{R}$ and $k \in \mathbb{N}$,

$$
\sum_{m=1}^{k} \frac{B^{m}}{m !} \sum_{i_{1}+\cdots+i_{m}=k} \frac{1}{i_{1} \cdots i_{m}}=\frac{B(B+1) \cdots(B+k-1)}{k !} .
$$

In particular, the sum on the left hand side vanishes if $B=-1$ and $k \geqslant 2$.

Proof: Let $\left[x^{k}\right] f(x)$ stand for the coefficient of $x^{k}$ in the Maclaurin series of $f(x)$. Since $\ln (1-x)^{-1}=$ $\sum_{i=1}^{\infty} \frac{x^{i}}{i}$, we have

$$
\left[x^{k}\right] \ln ^{m} \frac{1}{1-x}=\sum_{i_{1}+\cdots+i_{m}=k} \frac{1}{i_{1} \cdots i_{m}}
$$

${ }^{1}$ It follows that

$$
(1-x)^{-B}=\exp \ln (1-x)^{-B}=\sum_{m=0}^{\infty} \frac{B^{m}}{m !} \ln ^{m}(1-x)^{-1},
$$

${ }^{1}$ This identity is recorded in, for example, [3] due to its connection to combinatorics: $\frac{k !}{m !} \sum_{i_{1}+\cdots+i_{m}=k} \frac{1}{i_{1} \cdots i_{m}}=$ $\frac{1}{m !} \sum_{i_{1}+\cdots+i_{m}=k}\left(\begin{array}{c}k \\ i_{1} \cdots i_{m}\end{array}\right)\left(i_{1}-1\right) ! \cdots\left(i_{m}-1\right)$ ! = \# of ways to arrange $k$ objects into $m$ cycles, and these are called the (unsigned) Stirling numbers of the first kind [3]; (2.11) gives a generating function of these Stirling numbers. 


$$
\begin{aligned}
{\left[x^{k}\right](1-x)^{-B} } & =\left[x^{k}\right] \sum_{m=0}^{k} \frac{B^{m}}{m !} \ln ^{m}(1-x)^{-1}+\left[x^{k}\right] \sum_{m=k+1}^{\infty} \frac{B^{m}}{m !} \ln ^{m}(1-x)^{-1} \\
& =\sum_{m=0}^{k} \frac{B^{m}}{m !}\left[x^{k}\right] \ln ^{m}(1-x)^{-1}+0 \stackrel{(2.11)}{=} \sum_{m=1}^{k} \frac{B^{m}}{m !} \sum_{i_{1}+\cdots+i_{m}=k} \frac{1}{i_{1} \cdots i_{m}} .
\end{aligned}
$$

We finish the proof by noticing that $\left[x^{k}\right](1-x)^{-B}=B(B+1) \cdots(B+k-1) / k$ !

Proof of Proposition 2.3. By assumption, there is a $\bar{x}$ such that $\sum_{\ell=0}^{\bar{p}} a_{\ell} \pi(\ell)=\pi(\bar{x})$ for any polynomial $\pi(x)$ of degree no greater than $\bar{p}$. Since

$$
\left(\begin{array}{c}
x \\
j_{i_{1}+\cdots+i_{n-1}+1}
\end{array}\right) \cdots\left(\begin{array}{c}
x \\
j_{i_{1}+\cdots+i_{n}}
\end{array}\right)
$$

is a polynomial in $x$ of degree $\sum_{i=i_{1}+\cdots+i_{n-1}+1}^{i_{1}+\cdots+i_{n}} j_{i} \leqslant \sum_{i=1}^{k} j_{i} \leqslant \bar{p}$, we have

$$
\begin{aligned}
c_{j_{1} \cdots j_{k}} & =\sum_{\substack{1 \leqslant m \leqslant k \\
i_{1}+\cdots+i_{m}=k}} \frac{(-1)^{k-m}}{m ! i_{1} \cdots i_{m}}\left[\left(\begin{array}{c}
\bar{x} \\
j_{1}
\end{array}\right) \cdots\left(\begin{array}{c}
\bar{x} \\
j_{i_{1}}
\end{array}\right)\right] \cdots\left[\left(\begin{array}{c}
\bar{x} \\
j_{i_{1}+\cdots+i_{m-1}+1}
\end{array}\right) \cdots\left(\begin{array}{c}
\bar{x} \\
j_{k}
\end{array}\right)\right] \\
& =\left(\begin{array}{c}
\bar{x} \\
j_{1}
\end{array}\right) \cdots\left(\begin{array}{c}
\bar{x} \\
j_{k}
\end{array}\right) \sum_{m=1}^{k} \frac{(-1)^{k-m}}{m !} \sum_{i_{1}+\cdots+i_{m}=k} \frac{1}{i_{1} \cdots i_{m}} \\
& \stackrel{\text { Lemma } 2.4}{=} 0 .
\end{aligned}
$$

We also need Lemma 2.4 for bounding the size of the tail of $\sum_{k} T_{k}$. Let $\|\cdot\|$ be any matrix norm. By $(2.6)$

$$
\begin{aligned}
\left\|\sum_{k=K}^{\infty} T_{k}\right\| & =\left\|\sum_{k=K}^{\infty} \sum_{\substack{1 \leqslant m \leqslant k \\
i_{1}+\cdots+i_{m}=k}} \frac{(-1)^{k-m}}{m ! i_{1} \cdots i_{m}}\left(\sum_{\ell} a_{\ell} d_{\ell}^{i_{1}}\right) \cdots\left(\sum_{\ell} a_{\ell} d_{\ell}^{i_{m}}\right)\right\| \\
& \leqslant \sum_{k=K}^{\infty} \sum_{1 \leqslant m \leqslant k} \frac{1}{m !} \sum_{i_{1}+\cdots+i_{m}=k} \frac{1}{i_{1} \cdots i_{m}}\left(\sum_{\ell}\left|a_{\ell}\right|\left\|d_{\ell}\right\|^{i_{1}}\right) \cdots\left(\sum_{\ell}\left|a_{\ell}\right|\left\|d_{\ell}\right\|^{i_{m}}\right) \\
& \leqslant \sum_{k=K}^{\infty} \sum_{1 \leqslant m \leqslant k} \frac{1}{m !} \sum_{i_{1}+\cdots+i_{m}=k} \frac{C^{m}}{i_{1} \cdots i_{m}}\left[\max _{\ell}\left\|d_{\ell}\right\|\right]^{i_{1}+\cdots+i_{m}} \quad \text { where } C:=\sum_{\ell}\left|a_{\ell}\right| \\
& =\sum_{k=K}^{\infty}\left[\max _{\ell}\left\|d_{\ell}\right\|\right]^{k} \sum_{1 \leqslant m \leqslant k} \frac{C^{m}}{m !} \sum_{i_{1}+\cdots+i_{m}=k} \frac{1}{i_{1} \cdots i_{m}} \\
& \operatorname{Lemma} \sum_{k=K}^{\infty .4}\left[\max _{\ell}^{\infty}\left\|d_{\ell}\right\|\right]^{k} \frac{C(C+1) \cdots(C+k-1)}{k !} \\
& =\sum_{k=K}^{\infty}\left[\max _{\ell}\left\|d_{\ell}\right\|\right]^{k} \mathcal{O}(k\lceil\rceil-1) \\
= & \mathcal{O}\left(\left[\max _{\ell}\left\|d_{\ell}\right\|\right]^{K-\varepsilon}\right), \quad \forall \varepsilon>0 .
\end{aligned}
$$

Combining the above estimate and Proposition 2.3, we can obtain the following extrinsic proximity condition.

Proposition 2.5. Let $y: \mathbb{Z} \rightarrow G$ be such that $\max _{i}\left\|y_{i}\right\|<\infty$ and $\max _{i}\left\|y_{i}^{-1}\right\|<\infty .^{2}$ If $\bar{S}$ reproduces $\Pi_{0}$, then

$$
|S y-\bar{S} y|_{\infty}=\mathcal{O}\left(|\Delta y|_{\infty}^{2-\varepsilon}\right), \quad \forall \varepsilon>0 .
$$

\footnotetext{
${ }^{2}$ This condition is automatically satisfied if $G$ is compact, e.g. $O(n)$ or $S U(n)$.
} 
If $\bar{S}$ reproduces $\Pi_{\bar{p}}, \bar{p} \geqslant 2$, then

$$
|S y-\bar{S} y|_{\infty}=\mathcal{O}\left(|\Delta y|_{\infty}^{\bar{p}+1-\varepsilon}\right)+\mathcal{O}\left(\max \left|\Delta^{j_{1}} y\right|_{\infty} \cdots\left|\Delta^{j_{k}} y\right|_{\infty}\right), \quad \forall \varepsilon>0,
$$

where the maximum is taken over all $\left(j_{1}, \ldots, j_{k}\right)$ with $2 \leqslant k \leqslant \bar{p}, j_{1}+\cdots+j_{k} \geqslant \bar{p}+1$ and $1 \leqslant j_{i} \leqslant p$. (Recall that the mask $\left(a_{\ell}\right)$ is assumed to have a support of length $p+1$. This also implies that $\bar{p} \leqslant p$.)

Armed with this proximity condition, the "proximity $\Rightarrow$ smoothness" Theorem 2.2 implies that the nonlinear scheme $S$ produces limit curves $f: \mathbb{R} \rightarrow \mathbb{R}^{n \times n}$ with critical Hölder smoothness no less than that of the underlying linear scheme. Notice that, in the development of this section, we only assume that $G$ is immersed in $\mathbb{R}^{n \times n}$, so we cannot immediately claim that $f: \mathbb{R} \rightarrow G$ has the same smoothness as $f: \mathbb{R} \rightarrow \mathbb{R}^{n \times n}$. However, we have effectively proved Theorem 2.1 in the case when $G$ is a closed matrix group.

To rigorously prove that the same result holds for a not necessarily closed matrix group ${ }^{3}$, one needs to carefully argue that the subdivision algorithm itself, based on the 'intrinsic' log and exp maps of $G$ and an underlying convergent linear subdivision scheme, can guarantee that if one begins with a group of initial data points in $G$ close together to each other, then all the subdivided points at all scales are well-defined and continue to stay close to each other in the topology of $G$. This is basically step 1 . of the even more general argument in the next section, which deals with the case of $G$ being an arbitrary finite dimensionally Lie group.

\subsubsection{Extending the extrinsic proof to general Lie groups}

We discuss an argument by Wallner et al first used in [12] and subsequently in [13, 4, 5]. This argument begins with the Ado's theorem, which states that every Lie algebra is isomorphic to a matrix Lie algebra, which, in turns, implies that any Lie group $G$ is locally isomorphic to a matrix group $H \subset G L(n)$. There is no guarantee by the Ado's theorem that this $H$ is closed in $\mathbb{R}^{n \times n}$.

Since our subdivision scheme $S$ acts locally to the data, there is a minimal set of indices, which we denote here by $\mathcal{N}(0, i)$, such that the data $y_{0, k}, k \in \mathcal{N}(0, i)$, is enough to determine the limit function $S^{\infty} y$ at the whole interval $[i-1, i+1]$; of course it also means that $y_{j, k}$ can be determined for a finite range of indices $k$ at level $j$, we denote this set of indices by $\mathcal{N}(j, i)$. In fact,

$$
\mathcal{N}(j, i)=\left\{2^{j}(i-1)-M, \ldots, 2^{j}(i+1)+N\right\}
$$

where $M$ and $N$ are constants independent of $j$ but dependent on the support of the mask. For example, $M=N=2 k-2$ for the symmetric $2 k$-point Deslauriers-Dubuc scheme.

Wallner et al's idea is the following:

1. Argue that when we have a sequence of dense enough data points $\left(y_{0, k}\right)_{k \in \mathcal{N}(0, i)}$ in the matrix group $H$, then all the shifted subdivided points $y_{0, i}^{-1} y_{j, k}, k \in \mathcal{N}(j, i)$, are well-defined and stay in any pre-specified open neighborhood $U$ (in the topology of $H$ ) of the identity element $\mathbb{I}$ of $H$. By a basic shift invariance property of $S$, we can assume without loss of generality that $i=0$ and $y_{0,0}=\mathbb{I}$.

2. Saying $H$ and $G$ are locally isomorphic is equivalent to saying that there is a Lie algebra isomorphism $\psi: \mathfrak{h} \rightarrow \mathfrak{g}$ between their Lie algebras [10, Thm. 5, Ch. 10][6, Thm. 1.11, Ch. II]; moreover a local isomorphism can be chosen to be:

$$
\rho=\exp _{G} \circ \psi \circ \log _{H} .
$$

${ }^{3} \mathrm{~A}$ well-known example of a matrix group just immersed, but not embedded, in its ambient matrix space is given by:

$$
H:=\left\{\left[\begin{array}{cc}
R(x) & \mathbf{0} \\
\mathbf{0} & R(\sqrt{2} x)
\end{array}\right]: x \in \mathbb{R}\right\} \triangleleft G L(4), \text { where } R(x):=\left(\begin{array}{cc}
\cos 2 \pi x & -\sin 2 \pi x \\
\sin 2 \pi x & \cos 2 \pi x
\end{array}\right) .
$$

$H$, being a one-dimensional immersed submanifold of $\mathbb{R}^{4 \times 4}$, is not closed; the closure of $H$ in $\mathbb{R}^{4 \times 4}$ is the two-dimensional torus $\left\{\left[\begin{array}{cc}R(x) & \mathbf{0} \\ \mathbf{0} & R(y)\end{array}\right]: x, y \in \mathbb{R}\right\}$. 
(A subtlety: due to technical reasons, the proofs in both [10] and [6] do not directly involve this intuitive formula.) The reason of why (2.15) furnishes a local isomorphism can be seen from the Baker-Campbell-Hausdorff formula [11]:

$$
\exp (x) \exp (y)=\exp (x+y+1 / 2[x, y]+1 / 12[x,[x, y]]-1 / 12[y,[x, y]]+\cdots)
$$

holds for $x, y$ sufficiently close to 0 in a Lie algebra. The $\mathrm{BCH}$ formula tells us that (2.15) locally preserves the group operations in $G_{1}$ and $G_{2}$ exactly because $\psi$ preserves both the Lie brackets and the linear operations in $\mathfrak{g}_{1}$ and $\mathfrak{g}_{2}$.

3. Observe that subdivision by $S$ in $G$ is really the 'same' as subdivision by $S$ in $H$ when applied to data near the identity elements of the two Lie groups:

$$
\begin{aligned}
\rho\left(y_{j+1,2 k+1}\right) & =\rho\left(y_{j, k} R\left(y_{j, k}^{-1} y_{j, k-L}, \ldots, y_{j, k}^{-1} y_{j, k-L+p}\right)\right) \\
& =\rho\left(y_{j, k}\right) \rho\left(\exp _{H} \sum_{\ell=-L}^{-L+p} w_{\ell} \log _{H}\left(y_{j, k}^{-1} y_{j, k+\ell}\right)\right) \\
& =\rho\left(y_{j, k}\right) \exp _{G} \circ \psi \circ \log _{H}\left(\exp _{H} \sum_{\ell=-L}^{-L+p} w_{\ell} \log _{H}\left(y_{j, k}^{-1} y_{j, k+\ell}\right)\right) \\
& =\rho\left(y_{j, k}\right) \exp _{G}\left(\sum_{\ell=-L}^{-L+p} w_{\ell} \psi\left(\log _{H}\left(y_{j, k}^{-1} y_{j, k+\ell}\right)\right)\right) \\
& =\rho\left(y_{j, k}\right) \exp _{G}\left(\sum_{\ell=-L}^{-L+p} w_{\ell} \log _{G} \circ \exp _{G} \circ \psi\left(\log _{H}\left(y_{j, k}^{-1} y_{j, k+\ell}\right)\right)\right) \\
& =\rho\left(y_{j, k}\right) \exp _{G}\left(\sum_{\ell=-L}^{-L+p} w_{\ell} \log _{G}\left(\rho\left(y_{j, k}\right)^{-1} \rho\left(y_{j, k+\ell}\right)\right)\right) .
\end{aligned}
$$

This invariance property implies that as long as we can prove the smoothness property of $S$ in $H$, then we prove the whole Theorem 2.1.

\subsection{An Intrinsic Proof}

Let $G$ be any Lie group with the Lie algebra $\mathfrak{g}$. Let $\|\cdot\|$ be any norm in $\mathfrak{g}$. Let $\delta>0$ be small enough such that (i) if $U=\{a \in \mathfrak{g}:\|a\|<\delta\}$, then $y \mapsto e^{y}$ is a diffeomorphism from $U$ to its image and (ii) $f(a, b)=\log \left(e^{a} e^{b}\right)$ is smooth in $U \times U$. Define $g(a, b)=\log \left(e^{-a} e^{b}\right)$. Then $g$ is also smooth in $U \times U$. Note that

$$
f(a, 0)=a, \quad \text { and } \quad g(a, a)=0, \quad \forall a \in U .
$$

It follows that there exist $A, B \geqslant 1$ such that for any $a, b \in U$,

$$
\|g(a, b)\|=\|g(a, b)-g(a, a)\| \leqslant A\|b-a\|,
$$

and $\|f(a, b)-a\|=\|f(a, b)-f(a, 0)\| \leqslant B\|b\|$. So

$$
\|f(a, b)\| \leqslant\|a\|+B\|b\| .
$$

\subsubsection{Basic proximity condition and well-definedness of $S^{j} y$}

In the following, we show that if a sequence $y: \mathbb{Z} \rightarrow G$ is dense enough in the sense of

$$
y_{i}^{-1} y_{i+1} \in(\text { a small enough neighborhood of } e), \quad \forall i \in \mathbb{Z},
$$

then the subdivision scheme (1.2)-(1.3) is well-defined when applied to $y$ iteratively. Along the way, we also set up the basic machinery needed to prove the full proximity condition of the next section. 
First, we require $y$ to be at least dense enough so that for every $i \in \mathbb{Z}$,

$$
y_{i}^{-1} y_{i+\ell} \in \operatorname{dom}\left(\log _{G}\right) \quad \text { and } \quad x_{\ell}:=\log \left(y_{i}^{-1} y_{i+\ell}\right) \in U, \quad \forall \ell \in \mathcal{N}(0, i) .
$$

From now on, we work with the finite sequence $\left(x_{\ell}\right)$ in $U \subset \mathfrak{g}$ instead of the infinite sequence $y$ in $G$. Observe that (1.2)-(1.3) can be written locally in the Lie algebra as:

$$
(S x)_{2 k}=x_{k}, \quad(S x)_{2 k+1}=f\left(x_{k}, \sum_{\ell=-L}^{-L+p} w_{\ell} g\left(x_{k}, x_{k+\ell}\right)\right) ;
$$

this is well-defined as long as $\sum_{\ell} w_{\ell} g\left(x_{k}, x_{k+\ell}\right)$ stays in $U$, or

$$
\left\|\sum_{\ell} w_{\ell} g\left(x_{k}, x_{k+\ell}\right)\right\|<\delta .
$$

(We overload the notation $S$ and use it to represent also this rewritten version of the original $S$.) Since

$$
\begin{aligned}
\left\|\sum_{\ell} w_{\ell} g\left(x_{k}, x_{k+\ell}\right)\right\| & \leqslant \sum_{\ell}\left|w_{\ell}\right|\left\|g\left(x_{k}, x_{k+\ell}\right)\right\| \\
& \leqslant A \sum_{\ell}\left|w_{\ell}\right|\left\|x_{k+\ell}-x_{k}\right\| \\
& \leqslant A \sum_{\ell}\left|w_{\ell}\right||\ell|\|\Delta x\|_{\infty} \\
& \leqslant A N C\|\Delta x\|_{\infty},
\end{aligned}
$$

where $C:=\sum_{\ell}\left|w_{\ell}\right| \geqslant 1$ and $N=\max \{|\ell|: \ell \in \operatorname{support}(w)\}=\max (|-L|,|-L+p|)$. It follows that $S x$ is well-defined when

$$
\|x\|_{\infty}<\delta, \quad \text { and } \quad\|\Delta x\|_{\infty}<\frac{\delta}{A N C} .
$$

We need a stronger condition to guarantee that $S^{j} x$ is well-defined for all $j \geqslant 0$. For this purpose, we need to compare $S$ with the underlying linear scheme and then use a shrinking property of the linear scheme $((2.25))$.

Now, consider the linear subdivision operator $T$ defined by

$$
(T x)_{2 k}=x_{k}, \quad(T x)_{2 k+1}=\sum_{\ell} w_{\ell} x_{k+\ell} .
$$

Remark. We call this linear operator $T$, instead of $\bar{S}$, in order to distinguish it from the extrinsically defined $\bar{S}$ in the previous section. Note that $T$ operates on $\mathfrak{g}$-valued data, whereas $\bar{S}$ in the previous section operates on $\mathbb{R}^{n \times n}$-valued data. Of course, both schemes come from the same linear scheme applied componentwise to the relevant Euclidean spaces, so there cannot be any difference as far as smoothness is concerned; in particular, $s_{\infty}(T)=s_{\infty}(\bar{S})$.

In order to compare $S$ with $T$, consider:

$$
\begin{aligned}
(S x)_{2 k+1} & =f\left(x_{k}, \sum_{\ell} w_{\ell} g\left(x_{k}, x_{k+\ell}\right)\right) \\
& =f\left(x_{k}, 0\right)+\left.d f\right|_{\left(x_{k}, 0\right)}\left(\sum_{\ell} w_{\ell} g\left(x_{k}, x_{k+\ell}\right)\right)+O\left(\left\|\sum_{\ell} w_{\ell} g\left(x_{k}, x_{k+\ell}\right)\right\|^{2}\right) \\
& =f\left(x_{k}, 0\right)+\left.\sum_{\ell} w_{\ell} d f\right|_{\left(x_{k}, 0\right)}\left(g\left(x_{k}, x_{k+\ell}\right)\right)+O\left(\|\Delta x\|_{\infty}^{2}\right)
\end{aligned}
$$


and

$$
\begin{aligned}
(T x)_{2 k+1} & =\sum_{\ell} w_{\ell} x_{k+\ell} \\
& =\sum_{\ell} w_{\ell} f\left(x_{k}, g\left(x_{k}, x_{k+\ell}\right)\right) \\
& =\sum_{\ell} w_{\ell}\left[f\left(x_{k}, 0\right)+\left.d f\right|_{\left(x_{k}, 0\right)}\left(g\left(x_{k}, x_{k+\ell}\right)\right)+O\left(\left\|g\left(x_{k}, x_{k+\ell}\right)\right\|^{2}\right)\right] \\
& =f\left(x_{k}, 0\right)+\left.\sum_{\ell} w_{\ell} d f\right|_{\left(x_{k}, 0\right)}\left(g\left(x_{k}, x_{k+\ell}\right)\right)+\sum_{\ell} w_{\ell} O\left(\left\|x_{k+\ell}-x_{k}\right\|^{2}\right) \\
& =f\left(x_{k}, 0\right)+\left.\sum_{\ell} w_{\ell} d f\right|_{\left(x_{k}, 0\right)}\left(g\left(x_{k}, x_{k+\ell}\right)\right)+O\left(\|\Delta x\|_{\infty}^{2}\right),
\end{aligned}
$$

where $\left.d f\right|_{\left(x_{k}, 0\right)}$ denotes the differential of the function $f\left(x_{k}, \cdot\right): U \rightarrow \mathfrak{g}$ at 0 ; and (2.22)-(2.23) are simply the first order Taylor expansions of $f\left(x_{k}, \cdot\right)$ in action. Therefore

$$
\|S x-T x\|_{\infty}=O\left(\|\Delta x\|_{\infty}^{2}\right) .
$$

Assume $T$ is convergent. Then there exist $\bar{C}>0$ and $\bar{\mu}<1$ such that

$$
\left\|\Delta T^{j} x\right\|_{\infty} \leqslant \bar{C} \bar{\mu}^{j}\|\Delta x\|_{\infty}, \quad \forall j \in \mathbb{N} .
$$

By [14, Theorem 2.4], there exist $\delta^{\prime}>0, C^{\prime}>0$ and $\bar{\mu}<\mu<1$ such that when $\|\Delta x\|_{\infty}<\delta^{\prime}$ and $S^{j} x$ is well-defined, we have

$$
\left\|\Delta S^{j} x\right\|_{\infty} \leqslant C^{\prime} \mu^{j}\|\Delta x\|_{\infty} \leqslant C^{\prime}\|\Delta x\|_{\infty} .
$$

Since $S x$ is well-defined when $\|\Delta x\|_{\infty}<\frac{\delta}{A N C}$, it follows from (2.17) and (2.20) that

$$
\|S x\|_{\infty} \leqslant\|x\|_{\infty}+B A N C\|\Delta x\|_{\infty} .
$$

In general, if $S^{j} x$ is well-defined, then

$$
\begin{aligned}
\left\|S^{j} x\right\|_{\infty} & \leqslant\left\|S^{j-1} x\right\|_{\infty}+A B N C\left\|\Delta S^{j-1} x\right\|_{\infty} \\
& \leqslant \cdots \\
& \leqslant\|x\|_{\infty}+A B N C\left(\left\|\Delta S^{j-1} x\right\|_{\infty}+\cdots+\|\Delta x\|_{\infty}\right) \\
& \leqslant\|x\|_{\infty}+A B N C C^{\prime}\left(\mu^{j-1}+\cdots \mu^{0}\right)\|\Delta x\|_{\infty} \\
& \leqslant\|x\|_{\infty}+A B N C C^{\prime} \frac{1}{1-\mu}\|\Delta x\|_{\infty} .
\end{aligned}
$$

In order for $S^{j+1} x$ to be well-defined, we just need $\left\|S^{j} x\right\|_{\infty}<\delta$ and $\left\|\Delta S^{j} x\right\|_{\infty}<\delta / A N C$. Combining (2.27) and (2.26), we conclude that $S^{j+1} x$ exists for any $j \geqslant 0$ when

$$
\|x\|_{\infty}+\frac{A B N C C^{\prime}}{1-\mu}\|\Delta x\|_{\infty}<\delta, \quad \text { and }\|\Delta x\|_{\infty}<\min \left(\delta^{\prime}, \frac{\delta}{A N C C^{\prime}}\right) .
$$

Finally, since $\|\Delta x\|_{\infty} \leqslant 2\|x\|_{\infty}$, the above condition is satisfied if

$$
\|x\|_{\infty}<\min \left(\frac{\delta^{\prime}}{2}, \frac{\delta}{2 A N C C^{\prime}}, \frac{\delta}{1+\frac{2 A B N C C^{\prime}}{1-\mu}}\right)=: \varepsilon .
$$

If one wishes, one can translate the condition (2.28) in the above proposition to the condition (2.21), essentially by applying the exponential map to the ball $\{a \in \mathfrak{g}:\|a\|<\varepsilon\}$.

If for every $i \in \mathbb{Z}$ the finite sequence $\left(x_{\ell}\right)$ defined in (2.18) satisfies the condition (2.28), then the subdivision scheme $S$ as in (2.19) produces a well-defined limit curve on $\mathfrak{f}_{i}:[i-1, i+1] \rightarrow \mathfrak{g}$ for each $i$. Recall that the $S$ in (2.19) is really just the original scheme $S$ written locally in the Lie algebra, by exponentiating the $\mathfrak{f}_{\mathfrak{i}}$ 's and piecing them together, we conclude that the original scheme $S$ is well-defined and converge to a well-defined limit function $f: \mathbb{R} \rightarrow G$; in fact Theorem 2.2 theorem also tells us that $f$ is Hölder continuous. 


\subsubsection{Full proximity condition}

As in Proposition 2.5, suppose that the underlying linear interpolatory scheme $T$ reproduces $\Pi_{\bar{p}}$, so we have

$$
\sum_{\ell=-L}^{-L+p} w_{\ell} Q(\ell)=Q(1 / 2), \quad \forall Q \in \Pi_{\bar{p}}
$$

We shall use the Taylor's expansion of $f\left(x_{k}, \cdot\right): U \rightarrow \mathfrak{g}$ at 0 and that of $g\left(x_{k}, \cdot\right): U \rightarrow \mathfrak{g}$ at $x_{k}$. We write $\left.d^{m} f\right|_{\left(x_{k}, 0\right)}$ as the $m$-th derivative of $f\left(x_{k}, \cdot\right)$ at 0 and $\left.d^{m} g\right|_{\left(x_{k}, x_{k}\right)}$ as the $m$-th derivative of $g\left(x_{k}, \cdot\right)$ at $x_{k}$. By Taylor's theorem, we have

$$
\begin{aligned}
(S x)_{2 k+1}= & f\left(x_{k}, \sum_{\ell} w_{\ell} g\left(x_{k}, x_{k+\ell}\right)\right) \\
= & f\left(x_{k}, 0\right)+\left.\sum_{m=1}^{\bar{p}} \frac{1}{m !} d^{m} f\right|_{\left(x_{k}, 0\right)}\left(\sum_{\ell} w_{\ell} g\left(x_{k}, x_{k+\ell}\right), \cdots, \sum_{\ell} w_{\ell} g\left(x_{k}, x_{k+\ell}\right)\right)+O\left(\left\|\sum_{\ell} w_{\ell} g\left(x_{k}, x_{k+\ell}\right)\right\|^{\bar{p}+1}\right) \\
= & f\left(x_{k}, 0\right)+\left.\sum_{m=1}^{\bar{p}} \frac{1}{m !} \sum_{\ell_{1}, \cdots, \ell_{m}} w_{\ell_{1}} \cdots w_{\ell_{m}} d^{m} f\right|_{\left(x_{k}, 0\right)}\left(g\left(x_{k}, x_{k+\ell_{1}}\right), \cdots, g\left(x_{k}, x_{k+\ell_{m}}\right)\right)+O\left(\|\Delta x\|_{\infty}^{\bar{p}+1}\right) . \\
(T x)_{2 k+1} & =\sum_{\ell} w_{\ell} x_{k+\ell} \\
& =\sum_{\ell} w_{\ell} f\left(x_{k}, g\left(x_{k}, x_{k+\ell}\right)\right) \\
& =\sum_{\ell} w_{\ell}\left[f\left(x_{k}, 0\right)+\left.\sum_{m=1}^{\bar{p}} \frac{1}{m !} d^{m} f\right|_{\left(x_{k}, 0\right)}\left(g\left(x_{k}, x_{k+\ell}\right), \cdots, g\left(x_{k}, x_{k+\ell}\right)\right)+O\left(\left\|g\left(x_{k}, x_{k+\ell}\right)\right\|^{\bar{p}+1}\right)\right] \\
& =f\left(x_{k}, 0\right)+\left.\sum_{m=1}^{\bar{p}} \frac{1}{m !} \sum_{\ell} w_{\ell} d^{m} f\right|_{\left(x_{k}, 0\right)}\left(g\left(x_{k}, x_{k+\ell}\right), \cdots, g\left(x_{k}, x_{k+\ell}\right)\right)+O\left(\|\Delta x\|_{\infty}^{\bar{p}+1}\right) .
\end{aligned}
$$

So

$$
(S x)_{2 k+1}-(T x)_{2 k+1}=\sum_{m=2}^{\bar{p}} \frac{\theta_{m}-\bar{\theta}_{m}}{m !}+O\left(\|\Delta x\|_{\infty}^{\bar{p}+1}\right),
$$

where

$$
\begin{aligned}
\theta_{m} & =\left.\sum_{\ell_{1}, \cdots, \ell_{m}} w_{\ell_{1}} \cdots w_{\ell_{m}} d^{m} f\right|_{\left(x_{k}, 0\right)}\left(g\left(x_{k}, x_{k+\ell_{1}}\right), \cdots, g\left(x_{k}, x_{k+\ell_{m}}\right)\right), \\
\bar{\theta}_{m} & =\left.\sum_{\ell} w_{\ell} d^{m} f\right|_{\left(x_{k}, 0\right)}\left(g\left(x_{k}, x_{k+\ell}\right), \cdots, g\left(x_{k}, x_{k+\ell}\right)\right) .
\end{aligned}
$$

Since $g\left(x_{k}, x_{k}\right)=0$, it follows that

$$
g\left(x_{k}, x_{k+\ell}\right)=\left.\sum_{n=1}^{\bar{p}} \frac{1}{n !} d^{n} g\right|_{\left(x_{k}, x_{k}\right)}\left(x_{k+\ell}-x_{k}, \cdots, x_{k+\ell}-x_{k}\right)+O\left(\|\Delta x\|_{\infty}^{\bar{p}+1}\right) .
$$

Define

$$
D_{\ell^{\prime}}:=\sum_{j=0}^{\ell^{\prime}}(-1)^{\ell^{\prime}-j}\left(\begin{array}{l}
\ell \\
j
\end{array}\right) x_{k-L+j}, \quad \ell^{\prime}=0, \cdots, p .
$$

Then $x_{k-L+\ell^{\prime}}=\sum_{j=0}^{\ell^{\prime}}\left(\begin{array}{l}\ell \\ j\end{array}\right) D_{j}$ for $\ell^{\prime}=0, \cdots, p$. So for any $-L \leqslant \ell \leqslant-L+p$,

$$
x_{k+\ell}-x_{k}=\sum_{j=1}^{L+\ell}\left[\left(\begin{array}{c}
L+\ell \\
j
\end{array}\right)-\left(\begin{array}{c}
L \\
j
\end{array}\right)\right] D_{j}=\sum_{j \geqslant 1} A_{j}^{\ell} D_{j},
$$


where

$$
A_{j}^{\ell}:= \begin{cases}\left(\begin{array}{c}
L+\ell \\
j
\end{array}\right)-\left(\begin{array}{c}
L \\
j
\end{array}\right) & \text { if } 1 \leqslant j \leqslant L+\ell \\
0 & \text { otherwise }\end{cases}
$$

Hence

$$
\left.d^{n} g\right|_{\left(x_{k}, x_{k}\right)}\left(x_{k+\ell}-x_{k}, \cdots, x_{k+\ell}-x_{k}\right)=\left.\sum_{j_{1}, \cdots, j_{n}} A_{j_{1}}^{\ell} \cdots A_{j_{n}}^{\ell} d^{n} g\right|_{\left(x_{k}, x_{k}\right)}\left(D_{j_{1}}, \cdots, D_{j_{n}}\right) .
$$

Then

$$
\begin{aligned}
g\left(x_{k}, x_{k+\ell}\right) & =\left.\sum_{n=1}^{\bar{p}} \frac{1}{n !} \sum_{j_{1}, \cdots, j_{n}} A_{j_{1}}^{\ell} \cdots A_{j_{n}}^{\ell} d^{n} g\right|_{\left(x_{k}, x_{k}\right)}\left(D_{j_{1}}, \cdots, D_{j_{n}}\right)+O\left(\|\Delta x\|_{\infty}^{\bar{p}+1}\right) \\
& =\left.\sum_{n=1}^{\bar{p}} \frac{1}{n !} \sum_{J} Q_{J}(\ell) d^{n} g\right|_{\left(x_{k}, x_{k}\right)}\left(D_{J}\right)+O\left(\|\Delta x\|_{\infty}^{\bar{p}+1}\right)
\end{aligned}
$$

where

$$
J=\left(j_{1}, \cdots, j_{n}\right), \quad Q_{J}(\ell)=A_{j_{1}}^{\ell} \cdots A_{j_{n}}^{\ell}, \quad D_{J}=\left(D_{j_{1}}, \cdots, D_{j_{n}}\right)
$$

Therefore

$$
\left.d^{m} f\right|_{\left(x_{k}, 0\right)}\left(g\left(x_{k}, x_{k+\ell_{1}}\right), \cdots, g\left(x_{k}, x_{k+\ell_{m}}\right)\right)=\sum_{n_{1}, \cdots, n_{m}} \sum_{J_{1}, \cdots, J_{m}} \frac{Q_{J_{1}}\left(\ell_{1}\right) \cdots Q_{J_{m}}\left(\ell_{m}\right)}{n_{1} ! \cdots n_{m} !} F_{J_{1}, \cdots, J_{m}}^{n_{1}, \cdots, n_{m}}+O\left(\|\Delta x\|_{\infty}^{\bar{p}+1}\right),
$$

with

$$
F_{J_{1}, \cdots, J_{m}}^{n_{1}, \cdots, n_{m}}:=\left.d^{m} f\right|_{\left(x_{k}, 0\right)}\left(\left.d^{n_{1}} g\right|_{\left(x_{k}, x_{k}\right)}\left(D_{J_{1}}\right), \cdots,\left.d^{n_{m}} g\right|_{\left(x_{k}, x_{k}\right)}\left(D_{J_{m}}\right)\right) .
$$

Hence

$$
\theta_{m}=\sum_{n_{1}, \cdots, n_{m}} \sum_{J_{1}, \cdots, J_{m}} \frac{1}{n_{1} ! \cdots n_{m} !}\left[\sum_{\ell_{1}, \cdots, \ell_{m}} w_{\ell_{1}} \cdots w_{\ell_{m}} Q_{J_{1}}\left(\ell_{1}\right) \cdots Q_{J_{m}}\left(\ell_{m}\right)\right] F_{J_{1}, \cdots, J_{m}}^{n_{1}, \cdots, n_{m}}+O\left(\|\Delta x\|_{\infty}^{\bar{p}+1}\right) .
$$

Similarly, we have

$$
\bar{\theta}_{m}=\sum_{n_{1}, \cdots, n_{m}} \sum_{J_{1}, \cdots, J_{m}} \frac{1}{n_{1} ! \cdots n_{m} !}\left[\sum_{\ell} w_{\ell} Q_{J_{1}}(\ell) \cdots Q_{J_{m}}(\ell)\right] F_{J_{1}, \cdots, J_{m}}^{n_{1}, \cdots, n_{m}}+O\left(\|\Delta x\|_{\infty}^{\bar{p}+1}\right) .
$$

Note that for a fixed $j, A_{j}^{\ell}$ is a degree $j$ polynomial in $\ell$. So for fixed $J=\left(j_{1}, \cdots, j_{n}\right), Q_{J}(\ell)$ is a degree $|J|:=j_{1}+\cdots+j_{n}$ polynomial in $\ell$. Then it follows from (2.29) that when $\left|J_{1}\right|+\cdots+\left|J_{m}\right| \leqslant k$,

$$
\begin{aligned}
\sum_{\ell_{1}, \cdots, \ell_{m}} w_{\ell_{1}} \cdots w_{\ell_{m}} Q_{J_{1}}\left(\ell_{1}\right) \cdots Q_{J_{m}}\left(\ell_{m}\right) & =\left(\sum_{\ell} w_{\ell} Q_{J_{1}}(\ell)\right) \cdots\left(\sum_{\ell} w_{\ell} Q_{J_{m}}(\ell)\right) \\
& =Q_{J_{1}}\left(\frac{1}{2}\right) \cdots Q_{J_{m}}\left(\frac{1}{2}\right) \\
\sum_{\ell} w_{\ell} Q_{J_{1}}(\ell) \cdots Q_{J_{m}}(\ell) & =Q_{J_{1}}\left(\frac{1}{2}\right) \cdots Q_{J_{m}}\left(\frac{1}{2}\right) .
\end{aligned}
$$

Since for $J=\left(j_{1}, \cdots, j_{n}\right)$, we have

$$
\left\|\left.d^{n} g\right|_{\left(x_{k}, x_{k}\right)}\left(D_{J}\right)\right\|=O\left(\left\|D_{j_{1}}\right\| \cdots\left\|D_{j_{n}}\right\|\right)=O\left(\left\|\Delta^{j_{1}} x\right\|_{\infty} \cdots\left\|\Delta^{j_{n}} x\right\|_{\infty}\right) .
$$

So for $J_{1}=\left(j_{1}^{1}, \cdots, j_{n_{1}}^{1}\right), \cdots, J_{m}=\left(j_{1}^{m}, \cdots, j_{n_{m}}^{m}\right)$, we have

$$
\begin{aligned}
\left\|F_{J_{1}, \cdots, J_{m}}^{n_{1}, \cdots, n_{m}}\right\| & =O\left(\left\|\left.d^{n_{1}} g\right|_{\left(x_{k}, x_{k}\right)}\left(D_{J_{1}}\right)\right\| \cdots\left\|\left.d^{n_{m}} g\right|_{\left(x_{k}, x_{k}\right)}\left(D_{J_{m}}\right)\right\|\right) \\
& =O\left(\left\|\Delta^{j_{1}^{1}} x\right\|_{\infty} \cdots\left\|\Delta^{j_{n_{1}}^{1}} x\right\|_{\infty} \cdots\left\|\Delta^{j_{1}^{m}} x\right\|_{\infty} \cdots\left\|\Delta^{j_{n_{m}}^{m}} x\right\|_{\infty}\right) .
\end{aligned}
$$


Combining (2.36)-(2.40), we get

$$
\left\|\theta_{m}-\bar{\theta}_{m}\right\|=\sum_{\alpha_{1}+2 \alpha_{2}+\cdots+p \alpha_{p}>k} O\left(\|\Delta x\|_{\infty}^{\alpha_{1}} \cdots\left\|\Delta^{p} x\right\|_{\infty}^{\alpha_{p}}\right)+O\left(\|\Delta x\|_{\infty}^{\bar{p}+1}\right) .
$$

It then follows from (2.30) that

$$
\|S x-T x\|_{\infty}=\sum_{\alpha_{1}+2 \alpha_{2}+\cdots+p \alpha_{p}>k} O\left(\|\Delta x\|_{\infty}^{\alpha_{1}} \cdots\left\|\Delta^{p} x\right\|_{\infty}^{\alpha_{p}}\right)+O\left(\|\Delta x\|_{\infty}^{\bar{p}+1}\right) .
$$

As in the extrinsic proof, we can now invoke Theorem 2.2 to complete our intrinsic proof for Theorem 2.1.

\section{Variations}

\subsection{A modified scheme by Wallner/Nava Yazdani/Grohs}

In [13], Wallner et al consider an interesting modification of (2.2). This modification begins with the observation that one can rewrite an affine invariant linear subdivision scheme in the following way ${ }^{4}$ : Assume that the interpolatory mask $\left(w_{\ell}\right)_{\ell}$ is supported at $-L, \cdots,-L+p$ and also that $\sum_{\ell} w_{\ell}=1$, then

$$
(\bar{S} y)_{2 i+1}=\sum_{\ell=-L}^{-L+p} w_{\ell} y_{i+\ell}=y_{i}+\sum_{\ell=-L}^{-L+p-1} b_{\ell}\left(y_{i+\ell+1}-y_{i+\ell}\right)
$$

where $\left(b_{\ell}\right)_{\ell}$ is supported at $-L, \ldots,-L+p-1$ and

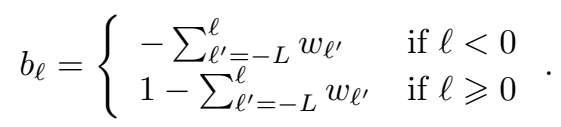

Then a nonlinear version of $\bar{S}$ for Lie-group valued data is defined to be:

$$
(W y)_{2 i+1}=y_{i} \exp \left(\sum_{\ell=-L}^{-L+p-1} b_{\ell} \log \left(y_{i+\ell}^{-1} y_{i+\ell+1}\right)\right) .
$$

In comparison with (2.2), the scheme $W$ only requires any two adjacent data points in the sequence $y$ to be closed in the sense of $y_{i}^{-1} y_{i+1} \in \widetilde{U}$ for all $i$, as opposed to $S$ which requires any $p$ consecutive data points in $y$ to be closed in the sense of $y_{i}^{-1} y_{i+\ell} \in \widetilde{U}$ for all $i$ and all $-L \leqslant \ell \leqslant-L+p$. From this point of view, Wallner et al's $W$ scheme appears to be a little more practical than Donoho et al's original $S$ scheme (2.2), although Wallner modestly commented that his original motivation for studying (3.3) instead of (2.2) was that the former scheme was more amendable to proving $C^{2}$.

One may wonder if our main Theorem 2.1 is true if $S$ is replaced by Wallner et al's $W$. The result in (3.3) does not answer this question; in fact it also does not answer the weaker question of whether $W$ based on a general $C^{2}$ linear interpolatory scheme $\bar{S}$ is also $C^{2}$.

However, it is very evident to us, through computational experiments, that Theorem 2.1 with $S$ replaced by $W$ is not true. We now present our computational evidences.

We randomly select a 5-periodic initial sequence $y_{0}: \mathbb{Z} \rightarrow S O(3)$ and subdivide it to level $j=9$ using both $S$ and $W$. The underlying linear interpolatory scheme $\bar{S}$ is chosen to be the 10-point DeslauriersDubuc scheme, with the mask $w_{1}=w_{0}=19845 / 32768, w_{2}=w_{-1}=-2205 / 16384, w_{3}=w_{-2}=567 / 16384$, $w_{4}=w_{-3}=-405 / 65536, w_{5}=w_{-4}=35 / 65536$, and is known to have smoothness $s_{\infty}(\bar{S})>4[16]$.

Below we plot the $(1,1)$-entries of $y_{9}$ from the two schemes, as well as their first, 2nd and 3rd divided differences. The limit function resulting from $W$ appears to be $C^{2}$ but not $C^{3}$, while that from $S$ appears to have a smooth third order derivative. Indeed, Theorem 2.1 says that in this case the limit function resulting from $S$ is $C^{4}$.

\footnotetext{
${ }^{4}$ Although we only deal with interpolatory schemes here, the idea works just as well for non-interpolatory schemes.
} 


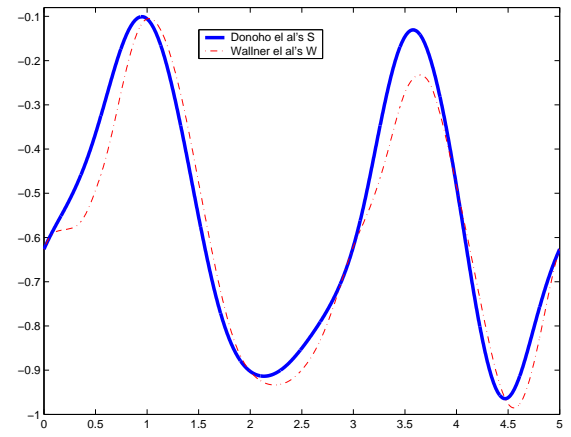

limit functions (plotted at level $j=9$ )

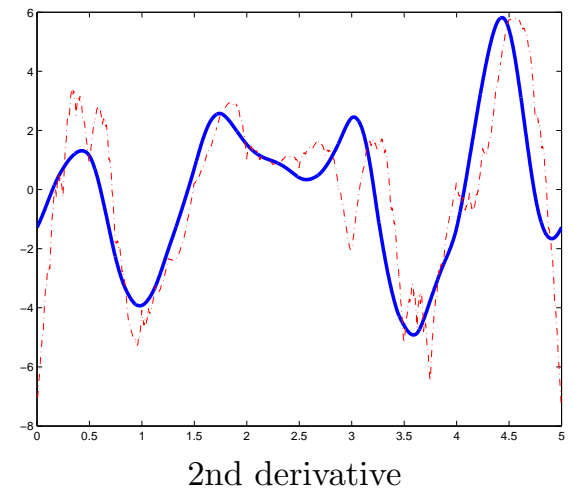

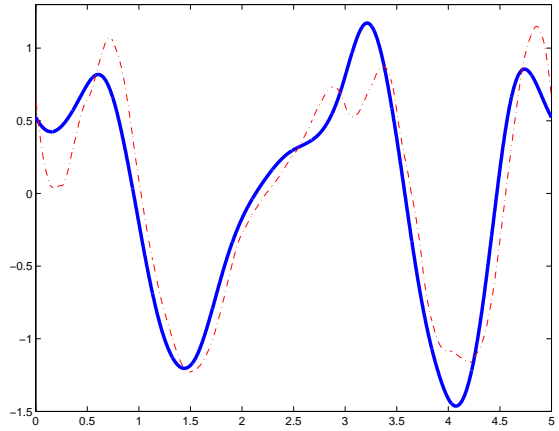

1st derivative

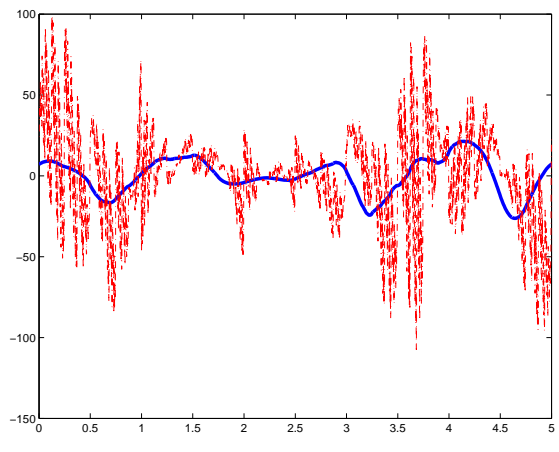

3rd derivative

Figure 1: Breakdown of Smoothness Equivalence for $W$

\subsection{Multivariate Extension}

It happens to be easy to extend the main proximity results in this paper to the setting where the domain of our Lie group-valued data is multivariate. We can pursue this in both the extrinsic and the intrinsic setting, for simplicity we only present the extrinsic case. Consider an $s$-dimensional interpolatory linear subdivision scheme operating on sequences $y: \mathbb{Z}^{s} \rightarrow \mathbb{R}$ :

$$
(\bar{S} y)_{2 \alpha+\epsilon}=\sum_{\nu} w_{\nu}^{\epsilon} y_{\alpha+\nu}, \quad \epsilon=\left(\epsilon_{1}, \ldots, \epsilon_{s}\right), \quad \epsilon_{i}=0,1
$$

with $w_{\nu}^{\mathbf{0}}=\delta_{\nu, \mathbf{0}}$. We say that $\bar{S}$ reproduces $\Pi_{\bar{p}}$ if

$$
\bar{S}\left(\left.\pi\right|_{\mathbb{Z}^{s}}\right)=\left.\pi\right|_{\frac{1}{2} \mathbb{Z}^{s}}, \quad \forall \pi \in \Pi_{\bar{p}},
$$

where $\Pi_{\bar{p}}$ is the space of $s$-variate polynomials of total degree not exceeding $\bar{p}$.

The associated $S$ operating on sequences $y: \mathbb{Z}^{s} \rightarrow G$ is given by

$$
(S y)_{2 \alpha+\epsilon}=y_{\alpha} \exp \left(\sum_{\nu} w_{\nu}^{\epsilon} \log \left(y_{\alpha}^{-1} y_{\alpha+\nu}\right)\right), \quad \epsilon=\left(\epsilon_{1}, \ldots, \epsilon_{s}\right), \quad \epsilon_{i}=0,1 .
$$

In the following derivation, $\alpha \in \mathbb{Z}^{s}$ and $\epsilon \neq \mathbf{0}$ are fixed and for all. We shall use standard multi-index notations.

Assume that the sequence $w^{\epsilon}$ is supported at $[-N, N]^{s}$. Let

$$
d_{\nu}:=y_{\alpha}^{-1} y_{\alpha+\nu-N}-\mathbb{I}, \quad \nu \in[0,2 N]^{s} .
$$

(In above $\alpha, \nu \in \mathbb{Z}^{s}$, and ' $\alpha+\nu-N$ ' means the vector $\alpha+\nu$ with all components subtracted by the integer $N$.) If we also write $a_{\nu}:=w_{\nu-N}^{\epsilon}$, then

$$
(S y)_{2 \alpha+\epsilon}=y_{\alpha} \exp \left(\sum_{0 \leqslant|\nu| \leqslant 2 N} a_{\nu} \log \left(\mathbb{I}+d_{\nu}\right)\right) .
$$


Similar to $(2.8)$, consider

$$
D_{\nu}:=\text { the } \nu \text {-th mixed difference of } d=\sum_{\mu \leqslant \nu}(-1)^{|\nu-\mu|}\left(\begin{array}{l}
\nu \\
\mu
\end{array}\right) d_{\mu}, \quad \nu \in[0,2 N]^{s} .
$$

The mapping of $\left(d_{\nu}\right)_{\nu \in[0,2 N]^{s}}$ to $\left(D_{\nu}\right)_{\nu \in[0,2 N]^{s}}$ is an invertible triangular linear mapping. Similar to (2.9), we can recover the $d_{\nu}$ 's from the $D_{\nu}$ 's by

$$
d_{\nu}=\sum_{\mu \leqslant \nu}\left(\begin{array}{l}
\nu \\
\mu
\end{array}\right) D_{\mu}, \quad \nu \in[0,2 N]^{s}
$$

As such, the extrinsic proximity condition derived in Section 2.2 almost goes through verbatim in the multivariate extension considered here. Similar to (2.5)-(2.10), we have

$$
y_{\alpha}^{-1}\left(S y_{2 \alpha+\epsilon}-\bar{S} y_{2 \alpha+\epsilon}\right)=\sum_{k=2}^{\infty} T_{k}
$$

where

$$
\begin{gathered}
T_{k}=\sum_{0 \leqslant\left|\mu_{1}\right|, \ldots,\left|\mu_{k}\right| \leqslant 2 N} c_{\mu_{1}, \ldots, \mu_{k}} D_{\mu_{1}} \cdots D_{\mu_{k}}, \\
c_{\mu_{1}, \ldots, \mu_{k}}=\sum_{\substack{1 \leqslant m \leqslant k \\
i_{1}+\cdots+i_{m}=k}} \frac{(-1)^{k-m}}{m ! i_{1} \cdots i_{m}}\left[\sum_{\nu} a_{\nu}\left(\begin{array}{c}
\nu \\
\mu_{1}
\end{array}\right) \cdots\left(\begin{array}{c}
\nu \\
\mu_{i_{1}}
\end{array}\right)\right] \cdots\left[\sum_{\nu} a_{\nu}\left(\begin{array}{c}
\nu \\
\mu_{i_{1}+\cdots+i_{m-1}+1}
\end{array}\right) \cdots\left(\begin{array}{c}
\nu \\
\mu_{k}
\end{array}\right)\right] .
\end{gathered}
$$

Now we have the following multivariate generalization of Proposition 2.3. If $\bar{S}$ reproduces $\Pi_{\bar{p}}$, then

$$
\left|\mu_{1}+\cdots \mu_{k}\right|=\left|\mu_{1}\right|+\cdots+\left|\mu_{k}\right| \leqslant \bar{p} \Longrightarrow c_{\mu_{1}, \ldots, \mu_{k}}=0 .
$$

The proof is that of Proposition 2.3 with superficial changes of univariate indices to multivariate ones; the key ideas are the identity in Lemma 2.4 and that

$$
\left(\begin{array}{c}
x \\
\mu_{i_{1}+\cdots+i_{n-1}+1}
\end{array}\right) \cdots\left(\begin{array}{c}
x \\
\mu_{i_{1}+\cdots+i_{n}}
\end{array}\right)
$$

is an $s$-variate polynomial in the variable $x=\left(x_{1}, \ldots, x_{s}\right)$ with total degree $\left|\mu_{i_{1}+\cdots+i_{n-1}+1}\right|+\cdots+\left|\mu_{i_{1}+\cdots+i_{n}}\right| \leqslant$ $\bar{p}$.

Using a multivariate version of the "proximity implies smoothness" theorem, we can extend Theorem 2.1 to the multivariate setting. See also [4].

\section{Credit where credit is due}

Essentially the same result was recently obtained by Grohs in [4]. Grohs offers a different proof which is also extrinsic in nature. The second author obtained the extrinsic proximity condition in Section 2.2 in November 2006, but found that certain technicalities in the Ado's theorem argument (see Section 2.2.1) too subtle to follow. Agonized by this situation, the authors worked out the intrinsic proof of the theorem in July 2007.

When the extrinsic proof was first developed in November 2006, the second author only worked in the univariate setting, and did not expect that the result could be easily extended to the multivariate setting. The result in Section 3.2 was obtained after the reading of [4]. We thank Philip Grohs for sharing his manuscripts as well as other unpublished results.

\section{References}

[1] G. Deslauriers and S. Dubuc. Symmetric iterative interpolation processes. Constructive Approximation, 5:49-68, 1989. 
[2] S. Dubuc. Interpolation through an iterative scheme. Journal of Mathematical Analysis and Applications, 114:185-204, 1986.

[3] R. L. Graham, D. E. Knuth, and O. Patashnik. Concrete Mathematics. Addison-Wesley Publishing Company, 1989.

[4] P. Grohs. Higher order smoothness of interpolatory multivariate subdivision in Lie groups. April 2007.

[5] P. Grohs and J. Wallner. Log-exponential analogues of univariate subdivision schemes in lie groups and their smoothness properties. 2007.

[6] S. Helgason. Differential Geometry, Lie Groups, and Symmetric Spaces. American Mathematical Society, 2001.

[7] A. Marthinsen. Interpolation in Lie groups. SIAM Journal on Numerical Analysis, 37(1):269-285, 1999.

[8] I. Ur Rahman, I. Drori, V. C. Stodden, D. L. Donoho, and P. Schröder. Multiscale representations for manifoldvalued data. Multiscale Modeling and Simulation, 4(4):1201-1232, 2005.

[9] T. Shingel. Interpolation in special orthogonal groups. Technical report, University of Cambridge, 2007. Available at http://www.damtp.cam.ac.uk/user/na/NA_papers/NA2007_06.pdf.

[10] M. Spivak. A comprehensive introduction to differential geometry, volume 1. Publish or Perish, Inc., 3rd edition, 2005.

[11] V. S. Varadarajan. Lie Groups, Lie Algebras and their Representations. Vol. 102 of Graduate Texts in Mathematics. Springer-Verlag, 1984.

[12] J. Wallner and N. Dyn. Convergence and $C^{1}$ analysis of subdivision schemes on manifolds by proximity. Computer Aided Geometric Design, 22(7):593-622, 2005.

[13] J. Wallner, E. Nava Yazdani, and P. Grohs. Smoothness properties of Lie group subdivision schemes. Multiscale Modeling and Simulation, 6(2):493-505, 2007.

[14] G. Xie and T. P.-Y. Yu. Smoothness equivalence properties of manifold-valued data subdivision schemes based on the projection approach. SIAM Journal on Numerical Analysis, 45(3):1200-1225, 2007.

[15] G. Xie and T. P.-Y. Yu. Smoothness equivalence properties of general manifold-valued data subdivision schemes. Multiscale Modeling and Simulation, XX(XX):XXX-XXX, 2008.

[16] T. P.-Y. Yu. On the regularity analysis of interpolatory Hermite subdivision schemes. Journal of Mathematical Analysis and Applications, 302(1):201-216, 2005. 